\title{
Materially-isospectral congruent membranes
}

\author{
H. P .W. Gottlieb*
}

(Received 6 October 2005; revised 18 July 2006)

\begin{abstract}
Materially-isospectral congruent membranes are membranes which have the same shape but different structure, that is different densities, yet they possess the same vibration frequency spectrum. Some approaches to finding such systems are described, and explicit formulae are presented for some annular and circular membranes. The results are numerically verified.
\end{abstract}

\section{Contents}

1 Introduction

C153

2 Annular membranes and radial power transformations 2.1 Example 1: Annular membrane with inverse 4th power density C157

*School of Science, Griffith University, Nathan, Queensland, Australia. mailto:H.Gottlieb@griffith.edu.au

See http://anziamj.austms.org.au/V47EMAC2005/Gottlieb for this article, (C) Austral. Mathematical Soc. 2006. Published August 1, 2006. ISSN 1446-8735 
3 Circular membranes and conformal mappings

3.1 Examples 2,3: Circular membranes isospectral to uniform membranes ...................... . . C162

3.2 Example 4: Circular membrane with linear radial density and corresponding isospectral membrane . . . . . . . . C162

4 Discussion

C164

References

C166

\section{Introduction}

Isospectral membranes are membranes having the same vibration frequency spectrum for given boundary condition. They may be shape-isospectral, differing in shape but being of uniform density, so one looks at eigenvalues of the Laplacian. In that case, the question [1] "Can one hear the shape of a drum?" arises, and the answer, at least for some shapes, is "No": there are uniform membranes of genuinely different shapes having the same characteristic frequency spectrum [2]. In this paper we look at the alternative problem of materially-isospectral membranes, which have the same shape (congruent) but have different material properties, in this case density functions. Here, "one cannot hear the density of a drum". There may be isospectrality for more than one boundary condition, such as fixed edges and free edges.

As well as being interesting in themselves as non-homogeneous systems with exact or related solutions, these examples have a practical side. For inverse problems, they indicate which sets of boundary conditions are not sufficient to distinguish the structure. They may be used as partial inputs to test inversion algorithms when comparing systems with some identical boundarycondition spectra. They also provide benchmark problems for testing the accuracy of numerical methods for solving partial differential equations in two dimensions with spatially varying equation coefficients. 
This paper deals with radial power transformations for annular membranes, leading to inverse-fourth-power densities isospectral with uniform membranes, and with conformal maps for circular membranes. Computed tables verify some examples.

\section{Annular membranes and radial power transformations}

For two-dimensional plane polar coordinates $(r, \theta)$, consider the radial power transformation

$$
r^{\prime}=\frac{R^{p+1}}{r^{p}}
$$

where $R$ is some length scale and $a \leq r \leq b$. For a non-trivial transformation $(p \neq-1)$, congruent annuli result only if $p=1$. Then with the choice $R^{2}=a b, r=a \Leftrightarrow r^{\prime}=b$ and $r=b \Leftrightarrow r^{\prime}=a$. (The inner and outer radii are interchanged, but still $a \leq r^{\prime} \leq b$.)

Thus we consider the inversion of coordinates

$$
r=\frac{a b}{r^{\prime}}, \quad \theta=\theta^{\prime}
$$

and relate functions

$$
u(r, \theta)=v\left(r^{\prime}, \theta^{\prime}\right) .
$$

Now if $v$ is the transverse displacement of vibrations of a homogeneous annular membrane with radii $a \leq r^{\prime} \leq b$, and with constant tension $T$ and constant density $\sigma_{0}$, then $v\left(r^{\prime}, \theta^{\prime}\right)$ satisfies a modified Helmholtz equation involving the two-dimensional Laplacian

$$
\nabla^{\prime 2} v=-\frac{\omega^{2}}{T} \sigma_{0} v
$$


where the prime refers to the primed coordinates and $\omega$ denotes the angular eigenfrequencies. With the transformation (2), the variable $u(r, \theta)$ is found [3] to satisfy the partial differential equation

$$
\nabla^{2} u=-\frac{\omega^{2}}{T} \sigma_{0} \frac{a^{2} b^{2}}{r^{4}} u
$$

This is the partial differential equation for the displacement of a congruent but inhomogeneous membrane with inverse fourth-power radial density

$$
\sigma(r)=\sigma_{0} \frac{a^{2} b^{2}}{r^{4}}
$$

and the same eigenfrequency spectrum $\{w\}$ which is therefore isospectral to the homogeneous (uniform) annular membrane with the same inner and outer radii. Note [3] that only if $p=1$ (or, trivially, -1 ) is the complete two-dimensional (radial and angular) Laplacian obtained as in (5).

Since $v=0 \Leftrightarrow u=0$, the condition of fixed rims (Dirichlet boundary condition (Dbc)) is preserved; and since $\frac{\partial v}{\partial r^{\prime}}=0 \Leftrightarrow \frac{\partial u}{\partial r}=0$, the condition of free rims (Neumann boundary condition $(\mathrm{Nbc})$ ) is preserved. Thus the membranes are isospectral in both Dbc and Nbc boundary configurations.

The vibration amplitude functions (that is, the eigenfunctions) are related by

$$
u_{n}^{m}(r, \theta)=v_{n}^{m}\left(\frac{a b}{r}, \theta\right),
$$

where the $v_{n}^{m}$ are the standard eigenfunctions for a homogeneous annular membrane with the appropriate boundary conditions [4, p.116]. They involve Bessel functions of the first and second kind of integer order $n$, and the trigonometric functions of order $n$. Here, $m$ denotes the $m$ th solution of the transcendental eigenvalue equation arising from the boundary conditions.

Interestingly, the membrane masses are equal. The mass of the uniform membrane is

$$
M_{\sigma_{0}}=\pi \sigma_{0}\left(b^{2}-a^{2}\right) .
$$


Whereas the mass of the inhomogeneous membrane is

$$
\begin{aligned}
M_{\sigma} & =2 \pi \int_{a}^{b} \frac{\sigma_{0} a^{2} b^{2}}{r^{4}} r d r \\
& =-\pi \sigma_{0} a^{2} b^{2}\left(\frac{1}{b^{2}}-\frac{1}{a^{2}}\right) \\
& =\pi \sigma_{0}\left(b^{2}-a^{2}\right) \\
& =M_{\sigma_{0}} .
\end{aligned}
$$

Since the inhomogeneous membrane (5) attains its maximum density at $r=a$ $\left(\sigma(a)=\sigma_{0} b^{2} / a^{2}\right)$ and its minimum density at $r=b\left(\sigma(b)=\sigma_{0} a^{2} / b^{2}\right)$, the density variation over this membrane is

$$
\frac{\sigma_{\max }}{\sigma_{\min }}=\frac{b^{4}}{a^{4}},
$$

which may be substantial, depending on the radius ratio.

More generally, if the $\left(r^{\prime}, \theta^{\prime}\right)$ membrane has a non-constant density $\sigma_{1}\left(r^{\prime}, \theta^{\prime}\right)$, and with $r=a b / r^{\prime}$, it turns out that there is isospectrality of $\sigma_{1}$ with

$$
\sigma_{2}(r, \theta)=\frac{a^{2} b^{2}}{r^{4}} \sigma_{1}\left(\frac{a b}{r}, \theta\right) .
$$

The masses are again equal:

$$
\begin{aligned}
M_{2} & =\int_{\theta=0}^{2 \pi} \int_{r=a}^{b} \sigma_{2}(r, \theta) r d r d \theta \\
& =\int_{\theta^{\prime}=0}^{2 \pi} \int_{r^{\prime}=b}^{a} \frac{r^{\prime 4}}{a^{2} b^{2}} \sigma_{1}\left(r^{\prime}, \theta^{\prime}\right) \frac{a b}{r^{\prime}}\left(-\frac{a b}{r^{\prime 2}}\right) d r^{\prime} d \theta^{\prime} \\
& =\int_{\theta^{\prime}=0}^{2 \pi} \int_{r^{\prime}=a}^{b} \sigma_{1}\left(r^{\prime}, \theta^{\prime}\right) r^{\prime} d r^{\prime} d \theta^{\prime}=M_{1} .
\end{aligned}
$$




\subsection{Example 1: Annular membrane with inverse 4th power density}

The results of some eigenvalue computations are presented in Tables 1 and 2, for annular membranes with $a=1 / 2, b=1, T=1, \sigma_{0}=1$, involving an inhomogeneous membrane with (6)

$$
\sigma(r)=\frac{1}{4 r^{4}} .
$$

Here, for the homogeneous membrane, $n$ signifies the number of nodal diameters, and $m-1$ is the number of (internal) nodal circles.

For these calculations, the "exact" angular frequencies were found numerically as the roots of the algebraic eigenvalue equation (obtained from the explicit eigenfunctions and boundary conditions) using Maple, and are therefore correct to the number of digits given. The "computed" eigenvalues $\left(\omega^{2}\right)$ were found using the Eigenmodes facility of the finite element PDE Toolbox package of MATLAB, for Dirichlet and for Neumann boundary conditions. For the Dirichlet case, Table 1, the first thirteen (including degenerate) sequential eigenvalues are listed, together with next circularly symmetric mode ( $n=0, m=2: 18$ th mode). The Uniform, Computed column exhibits, first of all, the degree to which degeneracies for the $n>0$ modes are recovered. Comparison with the Exact column then indicates an accuracy of about $0.1 \%$ when the numerical package with the 5th mesh (four refinements) was used. As expected, finite element methods over estimate the true values. The Computed values for the inverse 4 th power density follow the computed values for the uniform case very closely, to better than $0.1 \%$, and are convincing confirmation of the isospectrality. In this case, the ratio (10) assumes the value 16, so the variation in density over the domain of the inhomogeneous membrane is quite substantial. The agreement is thus all the more impressive, as the two membranes are very different in structure.

For the Neumann case, Table 2, the first 15 (including degenerate) nonzero sequential eigenvalues are listed (as well as the zero eigenvalue). In this 
TABLE 1: Eigenvalues $\omega^{2}$ for uniform and inverse-fourth-power density annular membranes, equation (6), with inner radius $a=0.5\left(T=1, \sigma_{0}=1, b=1\right)$ : Dirichlet boundary condition.

\begin{tabular}{ccccc}
\hline \multicolumn{4}{c}{ Uniform } & $\begin{array}{c}\text { Inverse 4th power (13) } \\
n\end{array}$ \\
$m$ & $\omega^{2}$ Exact & $\omega^{2}$ Computed & $\omega^{2}$ Computed \\
\hline 0 & 1 & 39.0133 & 39.0223 & 39.0223 \\
1 & 1 & 40.8725 & 40.8823 & 40.8824 \\
1 & 1 & 40.8725 & 40.8826 & 40.8827 \\
2 & 1 & 46.4285 & 46.4415 & 46.4418 \\
2 & 1 & 46.4285 & 46.4419 & 46.4428 \\
3 & 1 & 55.6179 & 55.6374 & 55.6391 \\
3 & 1 & 55.6179 & 55.6377 & 55.6396 \\
4 & 1 & 68.3388 & 68.3691 & 68.3731 \\
4 & 1 & 68.3388 & 68.3695 & 68.3740 \\
5 & 1 & 84.4569 & 84.5043 & 84.5127 \\
5 & 1 & 84.4569 & 84.5046 & 84.5142 \\
6 & 1 & 103.814 & 103.887 & 103.903 \\
6 & 1 & 103.814 & 103.888 & 103.906 \\
$\vdots$ & $\vdots$ & $\vdots$ & $\vdots$ & $\vdots$ \\
0 & 2 & 157.424 & 157.592 & 157.651 \\
\hline
\end{tabular}

case, because of the closer spacing and lower values, the 6th mesh was used for the finite element computation package, with consequent even more impressive confirmation of isospectrality. (The tabulated results well illustrate the appropriate isospectrality for the cases selected here and later, so it was not necessary to make more highly accurate numerical computations.) 
TABLE 2: Eigenvalues $\omega^{2}$ for uniform and inverse-fourth-power density annular membranes, equation (6), with inner radius $a=0.5\left(T=1, \sigma_{0}=1, b=1\right)$ : Neumann boundary condition.

\begin{tabular}{ccccc}
\hline \multicolumn{4}{c}{ Uniform } & $\begin{array}{c}\text { Inverse 4th power (13) } \\
\omega^{2} \text { Computed }\end{array}$ \\
\hline & $m$ & $\omega^{2}$ Exact & $\omega^{2}$ Computed & 0.0000 \\
1 & 1 & 1.83514 & 1.8352 & 1.8352 \\
1 & 1 & 1.83514 & 1.8352 & 1.8352 \\
2 & 1 & 7.18886 & 7.1891 & 7.1891 \\
2 & 1 & 7.18886 & 7.1891 & 7.1892 \\
3 & 1 & 15.6638 & 15.6645 & 15.6651 \\
3 & 1 & 15.6638 & 15.6645 & 15.6651 \\
4 & 1 & 26.7830 & 26.7845 & 26.7869 \\
4 & 1 & 26.7830 & 26.7845 & 26.7869 \\
5 & 1 & 40.1815 & 40.1845 & 40.1906 \\
5 & 1 & 40.1815 & 40.1846 & 40.1907 \\
0 & 1 & 40.8725 & 40.8748 & 40.8756 \\
1 & 2 & 43.0985 & 43.1012 & 43.1017 \\
1 & 2 & 43.0985 & 43.1013 & 43.1021 \\
2 & 2 & 49.8801 & 49.8842 & 49.8844 \\
2 & 2 & 49.8801 & 49.8842 & 49.8848 \\
\hline
\end{tabular}




\section{Circular membranes and conformal mappings}

Consider a region in the $x, y$-plane (with $z=x+i y \equiv r \exp i \theta$ ), and an analytic map $f$ to the $\xi, \eta$-plane:

$$
f(z)=\zeta=\xi+i \eta \equiv \rho \exp (i \phi),
$$

with $\xi=\xi(x, y)$ and $\eta=\eta(x, y)$. For a uniform (constant density $\sigma_{0}$ ) membrane (with unit tension $T=1$ ) in the $\xi, \eta$-plane, the amplitude function $v(\xi, \eta)$ satisfies the two-dimensional partial differential equation

$$
\nabla_{\xi, \eta}^{2} v+\omega^{2} \sigma_{0} v=0
$$

Let

$$
v(\xi, \eta)=u(x, y)
$$

Then it may be shown [5, Chapter 7] that, as a consequence of the CauchyRiemann equations, $u(x, y)$ satisfies the partial differential equation

$$
\nabla_{x, y}^{2} u+\omega^{2} \sigma_{0}\left|f^{\prime}(z)\right|^{2} u=0 .
$$

This shows that $u(x, y)$ is the amplitude function for an inhomogeneous membrane in the $x, y$-plane with density function

$$
\sigma(x, y)=\sigma_{0}\left|f^{\prime}(z)\right|^{2},
$$

with the same eigenfrequency spectrum.

We are interested here in circular membranes $[6,7]$. Up to rotations, the only one-to-one analytic mapping of the unit disc onto itself [5] is the conformal (Möbius) map

$$
\zeta=f(z)=\frac{z-\alpha}{1-\alpha z}, \quad-1<\alpha<1 .
$$


This has the property that $|\zeta|=1 \Leftrightarrow|z|=1$, so that the boundary is mapped onto itself. Furthermore, $v=0 \Leftrightarrow u=0$, so the fixed edge condition (Dbc) is preserved; and $\frac{\partial v}{\partial \rho}=0 \Leftrightarrow \frac{\partial u}{\partial r}=0$, so the free edge condition (Nbc) is also preserved.

Use of (19) in (18) yields the density function [6]

$$
\sigma(x, y)=\sigma_{0} \frac{\left(1-\alpha^{2}\right)^{2}}{\left[(1-\alpha x)^{2}+\alpha^{2} y^{2}\right]^{2}} .
$$

These constitute a family of densities, for $-1<\alpha<1$, of inhomogeneous (unit tension, unit radius) circular membranes which are isospectral to a standard homogeneous (unit radius) circular membrane, and to each other. This holds for both fixed and free boundary conditions.

More generally, if the membrane in the $\xi, \eta$-plane has non-uniform density $\sigma_{1}(\xi, \eta)$, then the mapped isospectral membrane in the $x, y$-plane has density function

$$
\sigma_{2}(x, y)=\left|f^{\prime}(z)\right|^{2} \sigma_{1}(\xi(x, y), \eta(x, y)) .
$$

The proof of equality of masses here uses the fact that the Jacobian of the transformation is just $\left|f^{\prime}(z)\right|^{2}$ :

$$
M_{1}=\iint \sigma_{1}(\xi, \eta) d \xi d \eta=\iint \sigma_{1}\left|f^{\prime}(z)\right|^{2} d x d y=\iint \sigma_{2} d x d y=M_{2} .
$$

The maximum density variation for the inhomogeneous membrane with density given by (20), which is materially-isospectral to a uniform membrane with constant density $\sigma_{0}$, is

$$
\frac{\sigma_{\max }(x=1, y=0)}{\sigma_{\min }(x=-1, y=0)}=\left(\frac{1+\alpha}{1-\alpha}\right)^{4}
$$

which may be appreciable for $\alpha$ near 1 . 


\subsection{Examples 2,3: Circular membranes isospectral to uniform membranes}

For a unit tension, unit radius circular membrane with constant, unit, density, the standard eigenfunction solutions involve Bessel functions [4], and hence the exact eigenvalues against which numerical finite element computations may be compared are available from the solutions of the resulting algebraic eigenfrequency equation $J_{n}(\omega)=0$ (for Dbc).

Table 3 lists the first ten values of $\omega^{2}$ for a uniform circular membrane (Dirichlet boundary condition) and for the isospectral non-uniform membranes with densities given by (20) for parameter values $\alpha=0.2$ and $\alpha=0.5$. For instance,

$$
\sigma_{\alpha=1 / 2}(x, y)=\frac{9}{\left[(2-x)^{2}+y^{2}\right]^{2}} .
$$

Again there is good correspondence. The ratio (23) has value 5.0625 for $\alpha=0.2$. For $\alpha=0.5$ the ratio is 81 , so there is a very large variation in density over the region. This may be reflected in the slightly less accurate values returned by the computational package. Agreement is nevertheless within about $0.1 \%$.

\subsection{Example 4: Circular membrane with linear radial density and corresponding isospectral membrane}

In this instance, two different non-uniform membranes isospectral to each other are considered, for parameter value $\alpha=1 / 2$. The first has a linear radial density

$$
\sigma_{1}=r
$$


TABLE 3: Eigenvalues $\omega^{2}$ for uniform circular membrane (Dirichlet boundary condition) and for densities (20) obtained by a conformal map, for values of the parameter $\alpha=1 / 5$ and $\alpha=1 / 2$.

\begin{tabular}{ccccc}
\hline & \multicolumn{2}{c}{ Uniform } & \multicolumn{2}{c}{ Conformal } \\
$n$ & $m$ & $\omega^{2}$ Exact & $\omega^{2}$ Computed & $\omega^{2}$ Computed \\
& & & $\alpha=0.2$ & $\alpha=0.5$ \\
\hline 0 & 1 & 5.7832 & 5.7835 & 5.7836 \\
1 & 1 & 14.6820 & 14.6839 & 14.6856 \\
1 & 1 & 14.6820 & 14.6840 & 14.6867 \\
2 & 1 & 26.3746 & 26.3810 & 26.3889 \\
2 & 1 & 26.3746 & 26.3812 & 26.3909 \\
0 & 2 & 30.4713 & 30.4796 & 30.4898 \\
3 & 1 & 40.7065 & 40.7219 & 40.7446 \\
3 & 1 & 40.7065 & 40.7223 & 40.7465 \\
1 & 2 & 49.2185 & 49.2389 & 49.2601 \\
1 & 2 & 49.2185 & 49.2416 & 49.2792 \\
\hline
\end{tabular}


and its isospectral partner via (21) and (19) has density

$$
\sigma_{2}=\frac{9}{16} \frac{\sqrt{\frac{1}{4}+x^{2}+y^{2}-x}}{\left[1+\frac{1}{4}\left(x^{2}+y^{2}\right)-x\right]^{5 / 2}} .
$$

This expression is clearly of non-radial form.

For a unit-tension, unit-radius circular membrane with linear radial density $r$ (25), it may be shown that, for fixed rim, the characteristic angular eigenfrequencies satisfy

$$
J_{\frac{2}{3} n}\left(\frac{2 \omega}{3}\right)=0 .
$$

Table 4 lists the first ten eigenvalues $\omega^{2}$ for comparison. The inhomogeneous density in equation (26) is rather complicated. Nevertheless, Table 4 exhibits good agreement with the simpler density (25), again to within about $0.1 \%$.

Amusingly, comparison of (27) with the eigenfrequency equation for the uniform membrane quoted in Section 3.1 above shows that, for $n=0$, the eigenvalues for the former are $(9 / 4)$ times those for the latter. This is born out by Tables 3 and 4 for $(n, m)=(0,1)$ and $(0,2)$. Other relationships for certain frequencies may be deduced. For instance, the $n=3$ eigenvalues obtained from (27) are (9/4) times those for the $n=2$ eigenvalues of the uniform membrane. This is demonstrated by the $m=1$ entries for those appropriate $n$ values in Tables 3 and 4 .

\section{Discussion}

This paper has demonstrated, by explicit constructions and numerical examples for annular and for circular membranes, the fact that "one cannot hear the structure of a drum", that is, "different densities can sound the same". This should be taken in the sense of Chapman [2] (stated there for different 
TABLE 4: Eigenvalues $\omega^{2}$ for unit radius circular membranes (Dirichlet boundary condition) with densities given by r (25) and by Equation (26).

$$
\sigma_{1}=r \quad \sigma_{2}(x, y)(26)
$$

\begin{tabular}{ccccc}
$n$ & $m$ & $\omega^{2}$ Exact & $\omega^{2}$ Computed & $\omega^{2}$ Computed \\
\hline 0 & 1 & 13.0122 & 13.0128 & 13.0130 \\
1 & 1 & 25.6382 & 25.6413 & 25.6449 \\
1 & 1 & 25.6382 & 25.6414 & 25.6481 \\
2 & 1 & 41.1267 & 41.1359 & 41.1521 \\
2 & 1 & 41.1267 & 41.1361 & 41.1560 \\
3 & 1 & 59.3429 & 59.3633 & 59.4059 \\
3 & 1 & 59.3429 & 59.3634 & 59.4094 \\
0 & 2 & 68.5603 & 68.5810 & 68.6269 \\
4 & 1 & 80.1958 & 80.2342 & 80.3173 \\
4 & 1 & 80.1958 & 80.2350 & 80.3299 \\
\hline
\end{tabular}

shapes) that they can produce exactly the same frequency spectrum. Thus for membranes with the same shape and the same boundary condition, a characteristic frequency spectrum may not be enough to distinguish whether they have the same density distribution. In particular, such considerations may have bearing on the determination of whether a system is uniform or has imperfections.

The addition of further information from a different boundary condition may still not lead to a resolution, as in the case of both Dirichlet (fixed rim) and Neumann (free rim) conditions above. Thus some other boundary condition, or some other property such as modal or nodal data [8], may have to be included if an inversion scheme to determine the density is to be successful.

All the isospectral examples in this paper were shown in each case to have equal mass. This suggests that knowledge of a frequency spectrum, whilst not allowing a determination of the actual density distribution of 
an inhomogeneous membrane of prescribed shape, might in general reveal the total mass. This is borne out by the considerations of Courant and Hilbert [9, p.442], where a cumulative eigenvalue counting function asymptotically yields $\iint \sigma d x d y$, that is, the mass of the membrane. An interesting question then arises of whether other broad structural features may also be obtainable from a frequency spectrum.

Acknowledgments: I thank Dr. Maeve McCarthy for discussions and hints on the use of MATLAB.

\section{References}

[1] M. Kac. Can one hear the shape of a drum? American Mathematical Monthly, 73 (4, Part 2):1-23, 1966. C153

[2] S. J. Chapman. Drums that sound the same. American Mathematical Monthly, 102:124-138, 1995. C153, C164

[3] H. P. W. Gottlieb. Axisymmetric isospectral annular plates and membranes. IMA Journal of Applied Mathematics, 49:185-192, 1992. http://dx.doi.org/10.1093/imamat/49.2.185 C155

[4] A. Gray, G. B. Mathews and T. M. MacRobert. A treatise on Bessel functions and their applications to physics (2nd edition). Dover, New York, 1966. C155, C162

[5] E. B. Saff and A. D. Snider. Fundamentals of Complex Analysis for Mathematics, Science and Engineering. Prentice-Hall, Englewood Cliffs, 1976. C160 
[6] H. P. W. Gottlieb. Density distribution for isospectral circular membranes. SIAM Journal on Applied Mathematics, 48:948-951, 1988. C160, C161

[7] H. P. W. Gottlieb. Isospectral circular membranes. Inverse Problems, 20:155-161, 2004. http://dx.doi.org/10.1088/0266-5611/20/1/009 C160

[8] G. M. L. Gladwell. Inverse Problems in Vibration (2nd edition). Kluwer, Dordrecht, 2004. C165

[9] R. Courant and D. Hilbert. Methods of Mathematical Physics, Volume I. Wiley, New York, 1953. C166 